\title{
Imaging the heliosheath using HSTOF energetic neutral atoms and Voyager 1 ion data
}

\author{
A. Czechowski ${ }^{1}$, M. Hilchenbach ${ }^{2}$, K. C. Hsieh $^{3}$, S. Grzedzielski ${ }^{1}$, and J. Kóta ${ }^{4}$ \\ 1 Space Research Center, Polish Academy of Sciences, Bartycka 18A, PL 00716 Warsaw, Poland \\ e-mail: ace@cbk.waw.pl \\ 2 Max-Planck-Institut für Sonnensystemforschung, Max-Planck-Strasse 2, 37191 Katlenburg-Lindau, Germany \\ e-mail: hilchenbach@mps .mpg.de \\ 3 Physics Department, University of Arizona, Tucson, AZ 85721, USA \\ e-mail: hsieh@physics . arizona.edu \\ ${ }^{4}$ Lunar and Planetary Laboratory, University of Arizona, Tucson, AZ 85721, USA \\ e-mail: kota@.lpl.arizona.edu
}

Received 11 February 2008 / Accepted 1 May 2008

\section{ABSTRACT}

\begin{abstract}
Context. Voyager 1 and 2 LECP instruments measure the distributions of the heliosheath ions of energies $\geq 40 \mathrm{keV}$. This threshold energy is an order of magnitude higher than the maximum energy $(6 \mathrm{keV})$ of the energetic neutral atoms (ENA) to be measured by the forthcoming IBEX mission. On the other hand, the energy range of SOHO/CELIAS/HSTOF ENA measurements is 58-88 keV for $\mathrm{H}$ and $28-58 \mathrm{keV} / n$ for He atoms, which overlaps with the energy range of Voyagers LECP. This offers a unique opportunity to combine HSTOF ENA measurements at 1 AU with LECP ion measurements in the heliosheath and obtain information about the large-scale structure of the heliosphere.

Aims. We use energetic neutral atoms data at $1 \mathrm{AU}$ and a Voyager 1 spectrum in the heliosheath to estimate the average column density of neutral hydrogen in selected sectors of the forward heliosheath.

Methods. We reanalyzed the energetic neutral hydrogen and helium data from HSTOF to identify the contribution to the neutral atoms flux originating in directions close to the apex of the Sun's motion relative to the local interstellar medium (LISM). We combine the data from HSTOF with the parent ion spectrum in the heliosheath measured by Voyager 1 to derive the background neutral hydrogen column density $N_{\mathrm{H}}$ in the heliosheath and estimate the thickness $L$ of the heliosheath within $\pm 45^{\circ}$ from the apex direction and in two $90^{\circ}$ wide flank sectors.

Results. In the forward sector of the heliosheath $N_{\mathrm{H}}=(0.63 \pm 0.19) \times 10^{14} \mathrm{~cm}^{-2}$, corresponding to the thickness $L=(42 \pm$ $12 \mathrm{AU}) / n_{\mathrm{H}} /\left(0.1 \mathrm{~cm}^{-3}\right)$ where $n_{\mathrm{H}}=$ average $\mathrm{H}$ density in the heliosheath. This is within the range of values predicted by theoretical models, but suggests that the heliosheath is thinner than expected. The hydrogen column densities in the flank sectors are not symmetric relative to the apex, but the difference is within the statistical uncertainty. The $\mathrm{H} / \mathrm{He}$ ratio measured by HSTOF is lower than the value following from Voyager 1 heliosheath spectra.
\end{abstract}

Key words. acceleration of particles - solar wind - interplanetary medium

\section{Introduction}

Since 1996, the HSTOF/CELIAS instrument onboard SOHO at $1 \mathrm{AU}$ has been surveying the ecliptic in energetic neutral hydrogen (ENH) (Hilchenbach et al. 1998, 2001) and energetic neutral helium (ENHe) (Czechowski et al. 2004). Hilchenbach et al. (1998) proposed that these energetic neutral atoms (ENA) are of heliospheric origin, produced by charge-exchange of the energetic ions in the distant heliosphere with the low energy neutral atoms that enter the heliosphere from the local interstellar medium (LISM). Because the energetic neutral atoms are only weakly coupled to plasma, they provide a useful method of remote sensing of the ion distributions in the distant heliosphere (Hsieh et al. 1992) and can be used for imaging the global structure of the heliosheath.

The energy range of HSTOF $(58-88 \mathrm{keV}$ for $\mathrm{H}$, $28-58 \mathrm{keV} / n$ for $\mathrm{He}$ ) is significantly above the energy (up to $6 \mathrm{keV}$ ) to be covered by the IBEX mission (McComas et al. 2004). In consequence, the parent ions of the ENAs observed by
HSTOF do not belong to the bulk solar wind or the pick-up ion population, but must be further accelerated, either by interacting with shocks or by plasma turbulence. The ions in the HSTOF ENA energy range are still not sufficiently energetic to penetrate far upstream from the source, against the solar-wind plasma flow. In particular, the ions accelerated at (or near) the termination shock are effectively confined to the region between the termination shock and the heliopause (the "inner heliosheath"). Also, the energetic "tails" of the pick-up ion distributions, although already present upstream of the termination shock, are strongly enhanced on crossing the termination shock into the heliosheath (Gloeckler \& Fisk 2006). The heliosheath is therefore probably a dominant source region of ENA in the HSTOF energy range.

The ENA flux arriving at the observation point in the inner solar system can in principle originate in any point along the line of sight (Hsieh et al. 1992). The ENH and ENHe fluxes observed by HSTOF must therefore include, along with the contribution from the heliosheath, contributions from the inner heliosphere, 
upstream from the termination shock. Identification of the different contributions has not been possible, because of the lack of detailed knowledge about the ion distributions in the heliosphere. After the Voyager 1 crossing of the termination shock, the heliosheath ion spectrum was measured for the first time (Decker et al. 2005; Stone et al. 2005). This permitted us to estimate the expected ENA flux from the heliosheath (Czechowski et al. 2006b, referred to as (I) in the following). We found that the neutral hydrogen flux from this source is of the same order of magnitude as the flux measured by HSTOF. Assuming that the observed hydrogen flux is dominated by the heliosheath contribution, we were able to estimate the average thickness of the part of the heliosheath covered by the HSTOF observations used in the study.

One shortcoming of that study was that, to reduce the statistical uncertainty, the ENA spectrum used in (I) was calculated from the HSTOF data averaged over a wide range of directions. The Voyager 1 trajectory, along which the ion spectra are measured, passes through the frontal part of the heliosheath, within $\sim 30^{\circ}$ from the apex of the Sun motion relative to the LISM (which we will call the "LISM apex" for brevity). The HSTOF data used in (I) included all observations along the line of sight within $155^{\circ}$ in ecliptic longitude from the LISM apex. To compare the ENA observations with the Voyager 1 data, we had therefore to assume for (I) that the ion spectrum measured by Voyager 1 could be used as an approximation of the average ion energy spectrum in the entire frontal portion (both forward and flanks) of the heliosheath.

We re-analysed the HSTOF hydrogen and helium data and derived the energy spectra of the ENA originating in the four $90^{\circ}$ wide sectors in ecliptic longitude chosen to cover separately the forward region (within $\pm 45^{\circ}$ from the LISM apex), the flanks and the heliotail (Hilchenbach et al. 2007). For the forward sector, we also obtained the ENA fluxes corresponding to six $15^{\circ}$ wide subsectors (Hilchenbach et al. 2006). The range in ecliptic latitude in each case was $\pm 17^{\circ}$, defined by the HSTOF's field of view. Although there remains a difference between the Voyager 1 position $\left(\sim 30^{\circ}\right.$ off the ecliptic) and the selected HSTOF observation regions, the difference is significantly reduced with respect to (I).

In this work, we combine Voyager 1 ion data and HSTOF ENA spectra derived from the re-analysed data to obtain the average column density $N_{\mathrm{H}}$ of neutral hydrogen in the forward sector of the heliosheath $\left( \pm 45^{\circ}\right.$ off the LISM apex $)$ and in two flank sectors. Although our results for the flank sectors are more uncertain than for the apex sector because of the larger distance from the Voyager 1 trajectory, we include them here to attempt "imaging" of the forward heliosphere.

For the forward sector, we find $N_{\mathrm{H}}=(0.63 \pm 0.19) \times$ $10^{14} \mathrm{~cm}^{-2}$, implying that the thickness of the heliosheath is equal to $(42 \pm 12 \mathrm{AU}) / n_{\mathrm{H}} /\left(0.1 \mathrm{~cm}^{-3}\right)$, where $n_{\mathrm{H}}$ is the average hydrogen density in the heliosheath. This is within the range of results from numerical models (see Sect. 4).

We confirm our previous observation (I) that the energetic neutral helium flux measured by HSTOF is higher (by a factor $\sim 2$ ) than expected from the helium ion flux measurements by Voyager 1.

The HSTOF and Voyager 1 observations and basic assumptions of our approach are presented in Sect. 2. Section 3 presents the fit results. A detailed discussion of the results is given in Sect. 4. The conclusions are summarized in Sect. 5.

\section{HSTOF and Voyager 1 observations}

HSTOF is a part of the CELIAS experiment (Hovestadt et al. 1995) onboard SOHO, launched in 1996 to the orbit around the L1 point. The instrument field of view is $\pm 2^{\circ}$ in and $\pm 17^{\circ}$ off the ecliptic, with the line of sight directed $37^{\circ}$ off the Sun. Until mid 2003, this direction was fixed (west off the Sun) and all ecliptic longitudes were scanned once a year. From mid 2003, the spacecraft has been reoriented twice a year and the LISM apex and antiapex sectors became inaccessible to HSTOF. The energy range of the ENA observation was $58-88 \mathrm{keV}$ for $\mathrm{H}$ and $28-58 \mathrm{keV} / n$ for He. The ENA observations were only possible during "quiet times" (low ion intensity). For this reason, a large part of the data comes from the period 1996-1997, close to a solar minimum. Because of low ENA fluxes, it is necessary to integrate the HSTOF data over long time periods, or equivalently over wide ranges of ecliptic longitude.

The Voyager 1 post-shock measurements (Decker et al. 2005; Stone et al. 2005) originate in the ecliptic longitude close to the LISM apex direction, $\sim 30^{\circ}$ ecliptic latitude, and the time period 2005-2007. The spectrum used in (I) corresponds to the first half of the year 2005. The LECP instrument onboard Voyager 1 has the lower energy limit of $40 \mathrm{keV}$ and overlaps with the ENA energy range of HSTOF. The lower energy channels of LECP do not distinguish between the different ion species ( $Z \geq 1$ ions), so that the He fraction can only be estimated from the higher energy channels.

Since the present understanding of the evolution of the ion distributions in the heliosheath is incomplete, we make the simplest assumption to be able to relate the HSTOF ENA data to Voyager 1 post-shock measurements: despite the difference in location and observation time, we assume that the ion distributions responsible for the selected HSTOF ENA data can be approximated by the ion spectra derived from Voyager 1 observations in the heliosheath.

To express the observed flux $J_{\mathrm{ENA}, i}(E)$ of the ENA species $i$ $\left(i=\mathrm{H}\right.$ or He) at energy $E$ in terms of the parent ions flux $J_{\text {ion, } i}(E)$ $\left(i=\mathrm{H}^{+}\right.$or $\left.\mathrm{He}^{+}\right)$in the source region, we use the following approximate equation (see also (I)):

$J_{\mathrm{ENA}, i}(E)=J_{\mathrm{ion}, i}(E) \sigma_{\mathrm{cx}, i, \mathrm{H}}(E) N_{\mathrm{H}}$.

Here $\sigma_{\mathrm{cx}, i, \mathrm{H}}(E)$ is the charge exchange cross section between the ion of the species $i$ and the background hydrogen (we use the fits given by Barnett et al. 1990), and $N_{\mathrm{H}}$ is the background hydrogen column density in the source region along the HSTOF line of sight. Production of the ENA by charge exchange with the background helium is not included, because the number density of helium in the forward heliosheath is expected to be small $\left(0.015 \mathrm{~cm}^{-3}\right.$ : Witte 2004) compared to the hydrogen density. The hydrogen density in the heliosheath is not well known (some estimations: Gloeckler et al. 1996: $0.115 \mathrm{~cm}^{-3}$; Quemerais et al. 1994: $0.165 \mathrm{~cm}^{-3}$ ). The losses to the ENA between the source and the observation point are small in the HSTOF energy range and therefore omitted from Eq. (1).

The ion spectrum $J_{\text {ion }, i}(E)$ we assume to be equivalent to the spectrum derived from Voyager 1 LECP observations in the heliosheath (Decker et al. 2005). The spectrum is the average over the first 160 days of Voyager 1 observations in the heliosheath. We assume that the average low energy spectrum does not change much along the Voyager 1 trajectory between the termination shock and the heliopause, which is found to be consistent with observations (Decker et al. 2006, 2007).

We note that while the HSTOF energy range overlaps with the LECP " $Z \geq 1$ ion" data, the LECP results with ion 
mass identified correspond to energies above the HSTOF range. Consequently, identification of the proton and helium contributions involves extrapolation from higher energy $(0.3 \mathrm{MeV} / n)$. We use the power-law fit $\left(J_{\text {fit }}(E) \propto E^{-1.67}\right)$ of Decker et al. (2005, see their Fig. 3) to the LECP combined ion data. The ion fluxes $J_{\text {ion, } i}(E)$ are assumed to be proportional to $J_{\text {fit }}(E)(E=$ total energy):

$J_{\text {ion, } \mathrm{H}}(E)=J_{\text {fit }}(E) / 0.6 \quad J_{\text {ion, } \mathrm{He}}(E)=0.672 J_{\text {fit }}(E)$.

The factor $1 / 0.6$ is the value given in Decker et al. (2005). The coefficient 0.672 provides the closest agreement with the helium ion flux data points in Fig. 3 of Decker et al. (see also (I)). We assume that Eq. (2) can be used for the sunwarddirected ion flux. This assumption is justified in view of the small ion flux anisotropy observed in the heliosheath (Decker et al. 2005, 2007).

The source region in Eq. (1) is assumed to be the heliosheath (between the termination shock and the heliopause). The contributions from regions other than the heliosheath should be subtracted from $J_{\mathrm{ENA}, i}$. Since these contributions can be estimated only approximately (see Sect. 4), we instead treat all HSTOF ENA flux as originating in the heliosheath. The results for $N_{\mathrm{H}}$ and $L$ must therefore be regarded as upper limits on the heliosheath values.

\section{Estimation of the hydrogen column density in the heliosheath}

We determine $N_{\mathrm{H}}$ by fitting the calculated ENA flux given by Eq. (1) to the observed ENA flux given by the HSTOF data. $N_{\mathrm{H}}$ is the only free parameter in the fit. The fit is performed by minimizing the quantity $\chi^{2}=\Sigma_{a}\left(J_{a}-(j \sigma)_{a} N_{\mathrm{H}}\right)^{2} / \Delta_{a}^{2}$ where $a$ denotes data points, $J_{a}$ represents the values of the ENA flux measured by HSTOF, $(j \sigma)_{a}$ is the product of the ion flux $J_{\text {ion, } i}(E)$ by the charge-exchange cross-section $\sigma_{\mathrm{cx}, i}(E)$ averaged over the HSTOF energy bin, and $\Delta_{a}$ are the statistical 1- $\sigma$ errors.

Apart from a fit to all $\mathrm{H}$ and He ENA data points, we also performed fits to restricted subsets of the data:

- fits using only $\mathrm{H}$ or only He data points. The motivation for "H only" fits was that the ion contamination of the helium data was probably larger than for the case of hydrogen. The "He only" fits were compared with "H only" fits to estimate the value of the $\mathrm{H}^{+} / \mathrm{He}^{+}$flux ratio in the source region, required to account for the HSTOF He data;

- fits excluding all but the lowest energy data points. The reason for this restriction was that the lowest energy HSTOF data points were likely to be least contaminated by the ion contribution.

We use the notation $\mathrm{H}(n)(\mathrm{He}(n))$ to describe the fit that includes $n$ hydrogen (helium) data points and $\mathrm{H}\left(n_{1}\right)+\mathrm{He}\left(n_{2}\right)$ for the simultaneous fit to $n_{1} \mathrm{H}$ and $n_{2}$ He data points.

Beside the fits to the ENA originating in the forward sector of the heliosheath $\left(210^{\circ}-300^{\circ}\right.$ ecliptic longitude), we completed similar fits to the data from the $90^{\circ}$ wide flank sectors: $120^{\circ}-210^{\circ}$ and $300^{\circ}-30^{\circ}$. It should be noted that in the flank sectors our model is less likely to be applicable than in the forward sector, if only due to larger distance from the Voyager 1 observation region, which implies that the use of the Voyager 1 spectrum is less justified.

The results of the fits are presented in Table 1. We note that the column density value corresponding to the " $\mathrm{H}+\mathrm{He}$ " fits is
Table 1. Fits to HSTOF ENA data using Voyager 1 ion spectrum.

\begin{tabular}{lccc}
\hline \hline Fit & sector & $N_{\mathrm{H}}\left(\mathrm{cm}^{-2}\right)$ & $\chi^{2}$ \\
\hline $\mathrm{H}(3)+\mathrm{He}(3)$ & $210^{\circ}-300^{\circ}$ & $(0.75 \pm 0.18) \times 10^{14}$ & 6.1 \\
$\mathrm{H}(1)+\mathrm{He}(1)$ & $210^{\circ}-300^{\circ}$ & $(0.66 \pm 0.22) \times 10^{14}$ & 0.74 \\
$\mathrm{H}(3)$ & $210^{\circ}-300^{\circ}$ & $(0.63 \pm 0.19) \times 10^{14}$ & 1.1 \\
$\mathrm{H}(1)$ & $210^{\circ}-300^{\circ}$ & $(0.55 \pm 0.25) \times 10^{14}$ & 0.0 \\
$\mathrm{He}(3)$ & $210^{\circ}-300^{\circ}$ & $(1.27 \pm 0.40) \times 10^{14}$ & 2.9 \\
$\mathrm{He}(1)$ & $210^{\circ}-300^{\circ}$ & $(0.99 \pm 0.45) \times 10^{14}$ & 0.0 \\
\hline $\mathrm{H}(3)+\mathrm{He}(3)$ & $120^{\circ}-210^{\circ}$ & $(0.86 \pm 0.16) \times 10^{14}$ & 6.8 \\
$\mathrm{H}(1)+\mathrm{He}(1)$ & $120^{\circ}-210^{\circ}$ & $(0.96 \pm 0.20) \times 10^{14}$ & 2.2 \\
$\mathrm{H}(3)$ & $120^{\circ}-210^{\circ}$ & $(0.76 \pm 0.18) \times 10^{14}$ & 2.4 \\
$\mathrm{H}(1)$ & $120^{\circ}-210^{\circ}$ & $(0.80 \pm 0.23) \times 10^{14}$ & 0.0 \\
$\mathrm{He}(3)$ & $120^{\circ}-210^{\circ}$ & $(1.25 \pm 0.37) \times 10^{14}$ & 2.9 \\
$\mathrm{He}(1)$ & $120^{\circ}-210^{\circ}$ & $(1.50 \pm 0.42) \times 10^{14}$ & 0.0 \\
\hline $\mathrm{H}(3)+\mathrm{He}(3)$ & $300^{\circ}-30^{\circ}$ & $(0.64 \pm 0.14) \times 10^{14}$ & 19.7 \\
$\mathrm{H}(1)+\mathrm{He}(1)$ & $300^{\circ}-30^{\circ}$ & $(0.66 \pm 0.17) \times 10^{14}$ & 3.5 \\
$\mathrm{H}(3)$ & $300^{\circ}-30^{\circ}$ & $(0.44 \pm 0.15) \times 10^{14}$ & 1.9 \\
$\mathrm{H}(1)$ & $300^{\circ}-30^{\circ}$ & $(0.51 \pm 0.19) \times 10^{14}$ & 0.0 \\
$\mathrm{He}(3)$ & $300^{\circ}-30^{\circ}$ & $(1.70 \pm 0.34) \times 10^{14}$ & 6.4 \\
$\mathrm{He}(1)$ & $300^{\circ}-30^{\circ}$ & $(1.31 \pm 0.38) \times 10^{14}$ & 0.0 \\
\hline
\end{tabular}

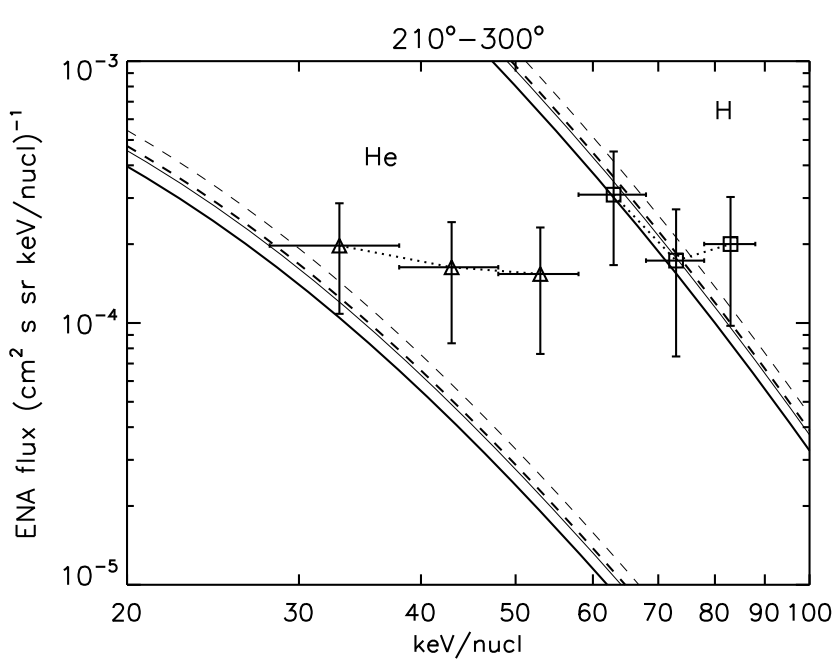

Fig. 1. The fits to the ENA data for the $90^{\circ}$ wide apex sector of the heliosheath (ecliptic longitude $210^{\circ}-300^{\circ}$; the LISM apex is at $\sim 254^{\circ}$ ). The solid lines show the fits to hydrogen data only: H(1) fit (the lowest energy point only, thick line) and $\mathrm{H}(3)$ (all three data points, thin line). The dashed lines show the simultaneous fits to $\mathrm{H}$ and $\mathrm{He}$ data: $\mathrm{H}(1)+\mathrm{He}(1)$ (only the lowest energy points, thick dashes) and $\mathrm{H}(3)+\mathrm{He}(3)$ (all data points, thin dashes) fits. See top section of Table 1.

close to the "H only" fits. The "He only" fits produce a significantly higher (by a factor $\sim 2$ ) $N_{\mathrm{H}}$.

Figures 1 and 2 show the ENA spectra that correspond to the fits ( $\mathrm{H}+\mathrm{He}$ and $\mathrm{H}$ only fits), compared with the HSTOF data. The agreement with the HSTOF $\mathrm{H}$ spectra is reasonably good for all three sectors. The fits to the ENA spectra pass below most of the $\mathrm{He}$ data points. This implies that the $\mathrm{H} / \mathrm{He}$ ENA flux ratio in the HSTOF data is lower than the $\mathrm{H} / \mathrm{He}$ ion flux ratio derived from Voyager 1 results. One possible explanation is that the observed He flux includes a contribution that cannot be attributed to the heliosheath He ions, which we discuss further in the next section. For this reason, we prefer the results derived from the " $\mathrm{H}$ only" fits $(\mathrm{H}(1)$ and $\mathrm{H}(3))$.

Figure 3 shows the average hydrogen column densities $N_{\mathrm{H}}$ in the forward and flank sectors of the heliosheath that are provided 

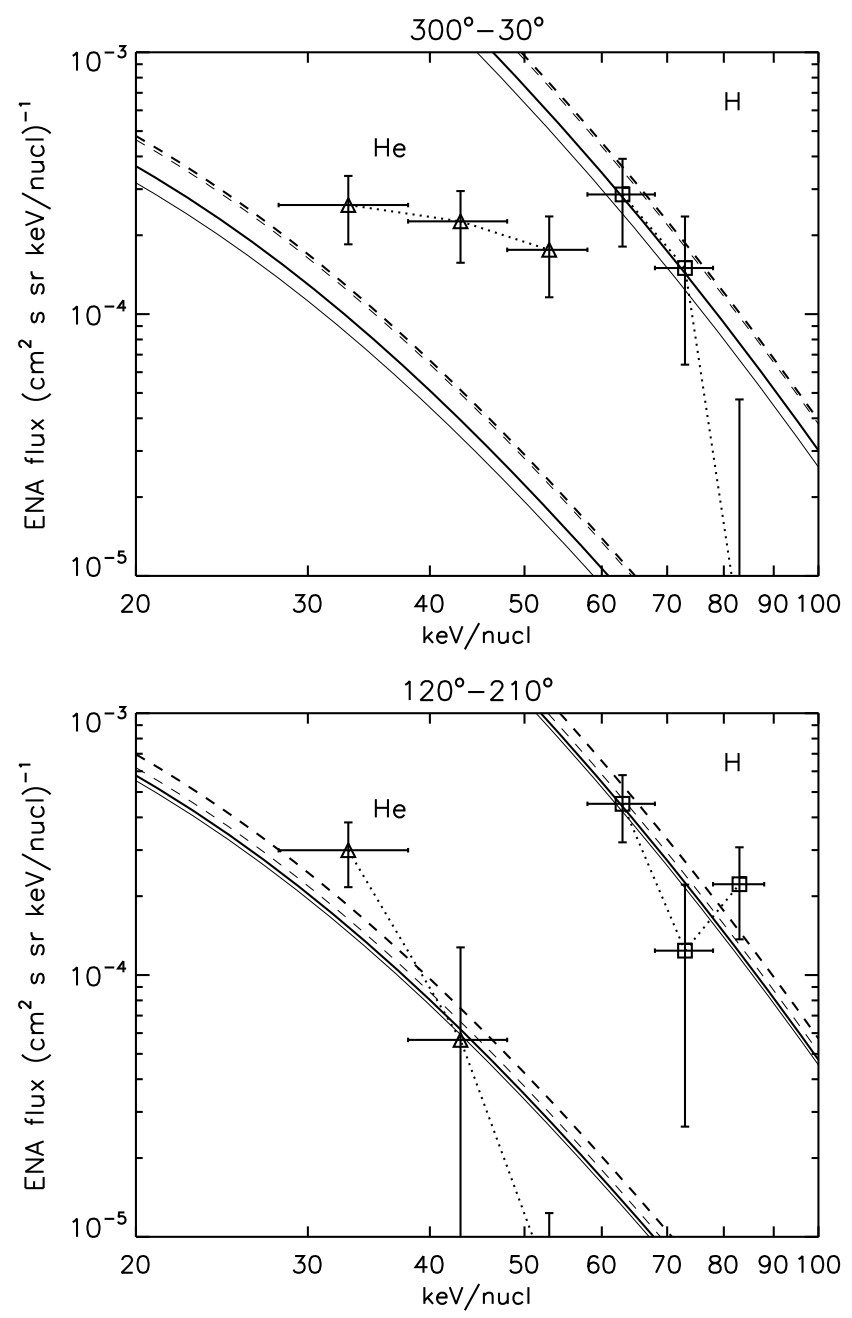

Fig. 2. As Fig. 1, but for the $90^{\circ}$ wide flank sectors of the heliosheath: ecliptic longitude $300^{\circ}-30^{\circ}$ and $120^{\circ}-210^{\circ}$. See bottom and middle section of Table 1.

by the $\mathrm{H}(1)$ fit (boxes), compared with the results from the models of the heliosphere corresponding to two cases of the Bonn model (Fahr et al. 2000) with different choices of the the neutral hydrogen and plasma densities in the LISM: model 1: $n_{\mathrm{H}, \mathrm{LISM}}=$ $0.1 \mathrm{~cm}^{-3}, n_{\mathrm{p}, \mathrm{LISM}}=0.1 \mathrm{~cm}^{-3}$ and model $2: n_{\mathrm{H}, \mathrm{LISM}}=0.2 \mathrm{~cm}^{-3}$, $n_{\mathrm{p}, \text { LISM }}=0.04 \mathrm{~cm}^{-3}$. For the models, the direction dependence of $N_{\mathrm{H}}$ is plotted. The $N_{\mathrm{H}}$ averages over the sectors are shown as horizontal intervals terminated by arrowheads.

\section{Discussion}

\subsection{Main results}

Our main result is the neutral hydrogen column density $N_{\mathrm{H}}$ in the forward sector $\left( \pm 45^{\circ}\right.$ ecliptic longitude from the LISM apex and $\pm 17^{\circ}$ latitude off the ecliptic) of the inner heliosheath. We consider the use of Voyager 1 ion spectrum in this sector to be more appropriate than in our previous study (I) where we had to extrapolate the Voyager data to a far wider sector. Our result: $N_{\mathrm{H}}=(0.63 \pm 0.19) \times 10^{14} \mathrm{~cm}^{-2}(\mathrm{H}(3) \mathrm{fit})$ corresponds to the thickness of the forward heliosheath $L=$ $(42 \pm 12 \mathrm{AU}) / n_{\mathrm{H}} /\left(0.1 \mathrm{~cm}^{-3}\right)$, where $n_{\mathrm{H}}$ is the average neutral hydrogen density in the forward heliosheath. This is similar to, but lower than most values derived from numerical models (see references cited in Table 2 and in Sect. 4.2). We note that this

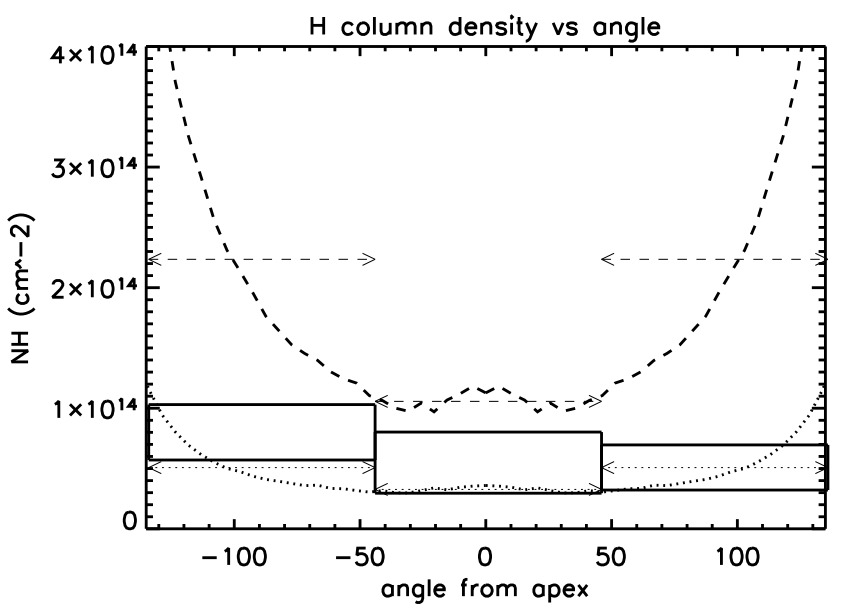

Fig. 3. Average column densities of neutral hydrogen (boxes) in the heliosheath forward and flank sectors, obtained from the $\mathrm{H}(1)$ fit to the HSTOF ENA data, compared to two gas-dynamical models (Fahr et al. 2000) of the heliosphere: model $1\left(n_{\mathrm{H}, \mathrm{LISM}}=0.1 \mathrm{~cm}^{-3}\right.$, $n_{\mathrm{p}, \mathrm{LISM}}=0.1 \mathrm{~cm}^{-3}$, dotted lines $)$ and model $2\left(n_{\mathrm{H}, \mathrm{LISM}}=0.2 \mathrm{~cm}^{-3}\right.$, $n_{\mathrm{p}, \mathrm{LISM}}=0.04 \mathrm{~cm}^{-3}$, dashed lines). For the models, the column density $N_{\mathrm{H}}$ is plotted as a function of direction, with the $N_{\mathrm{H}}$ averages over the sectors shown by horizontal line segments terminated by arrowheads (dashed or dotted, thin line). See Table 2.

result differs from our recent estimation (Czechowski et al. 2007) of $L$ (20-30 AU), which was based on independent analysis (Hilchenbach et al. 2006) of the HSTOF ENA data (the forward sector divided into $15^{\circ}$ longitude bins). For statistical reasons, we consider our present result to be better founded (no bins with small number of events).

The attempt to "image" the heliosheath is far more speculative, if only because of our assumption that the average ion flux in the flank sectors is the same as that for the forward sector. The derived values of $N_{\mathrm{H}}$ in the flank sectors are not symmetric with respect to the apex. Although this could be produced by the asymmetry of the heliosphere (a tilt towards larger ecliptic longitude?), we do not regard this result as conclusive due to uncertainties in the data and simplifications in the model.

\subsection{Comparison with models}

All models considered are two-dimensional (axisymmetric with respect to the LISM flow direction relative to Sun) and timeindependent, so that they do not include the effects of timedependence and three-dimensional structure of the heliosphere. In reality, the three-dimensional structure of the heliosphere, which is caused in particular by the anisotropic pressure of the interstellar magnetic field and the solar wind dependence on heliolatitude, and its time variation, due to the solar cycle and transient variations in the solar wind, are likely to be pronounced, and some are taken into account in the simulations, particularly the later results (a concise review is given by Pogorelov et al. 2008a; see also Izmodenov \& Alexashov 2006; Izmodenov et al. 2008; Pogorelov et al. 2008b; Washimi et al. 2007). We selected the simpler models because they cover a comparatively wide range of the parameters of the LISM, and because the information needed to estimate $N_{\mathrm{H}}$ was available for them.

Results from selected numerical models of the heliosphere are listed in Table 2. The values of $N_{\mathrm{H}}$, which were not given in the original papers, were estimated using the $\mathrm{H}$ profiles or, if these were not available, the values of $L$ and $n_{\mathrm{H}, \mathrm{TS}}$, the $\mathrm{H}$ density at the termination shock, together with the 
Table 2. Results from numerical models.

\begin{tabular}{lcccc}
\hline \hline Model & $\begin{array}{c}n_{\mathrm{p}, \text { LISM }} \\
\left(\mathrm{cm}^{-3}\right)\end{array}$ & $\begin{array}{c}n_{\mathrm{H}, \mathrm{LISM}} \\
\left(\mathrm{cm}^{-3}\right)\end{array}$ & $\begin{array}{c}L \text { (apex }) \\
(\mathrm{AU})\end{array}$ & $\begin{array}{c}N_{\mathrm{H}}(\text { apex }) \\
\left(\mathrm{cm}^{-2}\right)\end{array}$ \\
\hline $1^{a}$ & 0.1 & 0.1 & 39 & $0.36 \times 10^{14}$ \\
$2^{a}$ & 0.04 & 0.2 & 45 & $1.1 \times 10^{14}$ \\
$3^{b}$ & 0.04 & 0.24 & 47 & $1.1 \times 10^{14}$ \\
$4^{b}$ & 0.1 & 0.1 & 33 & $0.23 \times 10^{14}$ \\
$5^{b}$ & 0.1 & 0.14 & 25 & $0.21 \times 10^{14}$ \\
$6^{b}$ & 0.1 & 0.24 & 27 & $0.44 \times 10^{14}$ \\
$7^{c}$ & 0.1 & 0.2 & 55 & $0.9 \times 10^{14}$ \\
$8^{c}$ & 0.07 & 0.2 & 75 & $1.3 \times 10^{14}$ \\
$9^{c}$ & 0.04 & 0.2 & 85 & $1.6 \times 10^{14}$ \\
\hline
\end{tabular}

${ }^{a}$ Bonn model: Fahr et al. (2000); ${ }^{b}$ multifluid: Müller et al. (2006); ${ }^{c}$ kinetic: Izmodenov et al. (1999).

relation $N_{\mathrm{H}}=1.2 L n_{\mathrm{H}, \mathrm{TS}}$. The models can be divided into three classes, which differ in the method of describing the neutral component, that is single-fluid (the Bonn model: Fahr et al. 2000; Scherer \& Fahr 2003), multifluid (Zank et al. 1996 and others) or kinetic (Baranov \& Malama 1993 and others), the last being preferable for physical reasons (large mean free path for neutral atom-plasma coupling). There is a systematic difference between the results: in particular, the "kinetic" models predict a higher value of $n_{\mathrm{H}}$ in the forward heliosheath (Alexashov \& Izmodenov 2005; Heerikhuisen et al. 2006; Müller et al. 2007).

The picture of the heliosheath that is produced by the models depends on the assumed parameters of the LISM. There are arguments that propose high neutral $\mathrm{H}$ density values for the LISM $\left(\sim 0.2 \mathrm{~cm}^{-3}\right)$. Such values are implied by the ionization models of the local interstellar medium $\left(n_{\mathrm{H}, \mathrm{LISM}}=0.19-0.2 \mathrm{~cm}^{-3}\right.$ : Slavin \& Frisch 2007) and, independently, by the estimates of the $\mathrm{H}$ density close to the termination shock $\left(\sim 0.1 \mathrm{~cm}^{-3}\right)$ following from the pick-up ion production rate (Gloeckler et al. 1996) and the Lyman- $\alpha$ scattering observations (Quemerais et al. 1994). The electron or proton density are more poorly constrained: for example, the models of Slavin and Frisch (2007) imply $n_{\mathrm{e}, \mathrm{LISM}}=0.05-0.08 \mathrm{~cm}^{-3}$.

Table 2 shows that our result $\left(N_{\mathrm{H}}=(0.63 \pm 0.19) \times 10^{14} \mathrm{~cm}^{-2}\right)$ is in between the values derived using the models 6 and 7 , which both correspond to high values of $n_{\text {H,LISM }}\left(0.24\right.$ or $\left.0.2 \mathrm{~cm}^{-3}\right)$ and a high proton density $n_{\mathrm{p}, \mathrm{LISM}}=0.1 \mathrm{~cm}^{-3}$. Model 6 is of the multi-fluid type and may therefore underestimate $N_{\mathrm{H}}$ in comparison to kinetic models such as model 7.

We conclude that our result is consistent with numerical models for acceptable values of the LISM parameters $\left(n_{\mathrm{p}, \mathrm{LISM}} \sim\right.$ $0.1 \mathrm{~cm}^{-3}, n_{\text {H,LISM }} \sim 0.2 \mathrm{~cm}^{-3}$ ). We note, however, that some calculations imply that a lower value of $n_{\mathrm{p}}\left(\sim 0.05 \mathrm{~cm}^{-3}\right)$ is required to explain the observed termination shock distance (Pogorelov et al. 2008a). Also, if a significant part of the HSTOF ENA flux does not originate in the heliosheath, the heliosheath $N_{\mathrm{H}}$ would be smaller than our value. It may then be difficult to obtain an agreement with the models for the preferred (high) value of neutral $\mathrm{H}$ density in the LISM $\left(\sim 0.2 \mathrm{~cm}^{-3}\right)$ (see Sect. 4.4 for further discussion).

\subsection{Helium}

The helium ENA data cannot be reproduced as well by the model as hydrogen data. Some excess of helium remains in the data with respect to the model predictions. The value of $N_{\mathrm{H}}$ obtained from the fits to the hydrogen ENA data points (excluding helium) corresponds to the helium ENA flux that is below the lowest energy helium data point by a factor of approximately 2. Fitting of the helium ENA data points (excluding hydrogen) produce $N_{\mathrm{H}}$ that is too high by a similar factor.

As pointed out in (I), the helium ENA measurement provided by HSTOF data may overestimate the actual ENA flux due to contamination by helium ions. This is most likely to occur for the higher energy data points.

On the other hand, the value of the $\mathrm{H} / \mathrm{He}$ ion flux ratio $(\sim 10)$ derived from the HSTOF data is close to the value $(\sim 10)$ expected from the ACR modulation models rather than the value ( 20) observed by Voyager 1 downstream of the shock (Stone et al. 2005). The HSTOF helium data could be explained if the $\mathrm{H} / \mathrm{He}$ ion ratio became lower $(\sim 10)$ further downstream from the termination shock, since the ENA data are sensitive to ion fluxes averaged over large distances. However, there was no change in the $\mathrm{H} / \mathrm{He}$ ratio reported for Voyager 1 observations (Stone et al. 2007).

There is also a possibility that a significant portion of the observed helium ENA originates in a (so far unknown) source, which is not located in the inner heliosheath; this source would have to be characterized by low $\mathrm{H} / \mathrm{He}$ production ratio.

\subsection{Contribution from the inner heliosphere}

HSTOF ENA data must include contributions from regions other than the inner heliosheath, such as the region beyond the heliopause (including the outer heliosheath and the interstellar medium) and the inner heliosphere (the region inside the solar wind termination shock).

The first of those contributions would originate in the low energy part of the galactic cosmic ray (GCR) spectrum and from ions accelerated in the heliosheath. The GCR contribution is probably low $\left(\sim 3 \times 10^{-6}\left(\mathrm{~cm}^{2} \mathrm{~s} \mathrm{sr} \mathrm{kev}\right)^{-1}\right.$ : Czechowski et al. 2006a). Since the ion acceleration is probably more effective in the inner than the outer heliosheath, the corresponding contribution depends on the probability of passage of the accelerated ions across the heliopause.

In the inner heliosphere, the ENA in the HSTOF energy range are produced by neutralization of the ions accelerated in the solar wind, either by transient shock or by turbulence. Energetic "tails" are indeed observed in the ion distributions (Gloeckler \& Fisk 2006).

The most reliable method to estimate this contribution would be to use the accelerated ion data collected by Voyager LECP. Unfortunately, the published information is insufficient to reconstruct the evolution of the energetic ion spectrum along the Voyagers' orbits.

Kóta et al. (2001) estimated the production of hydrogen ENA by ions accelerated by the corotating interaction regions (CIRs). The method combined the model of CIR evolution with the observed accelerated ion spectrum close to the selected CIR shock. The calculated flux was about one order of magnitude too low to explain the peak HSTOF fluxes. However, close to the LISM apex direction, the CIR ENA flux at $70 \mathrm{keV}$ was $\sim 0.8-1.5 \times$ $10^{-4}\left(\mathrm{~cm}^{2} \mathrm{~s} \mathrm{sr} \mathrm{keV}\right)^{-1}$, which is comparable to the HSTOF apex flux in the $68-78 \mathrm{keV}$ interval of $1.4 \times 10^{-4}\left(\mathrm{~cm}^{2} \mathrm{~s} \mathrm{sr} \mathrm{keV}\right)^{-1}$.

Voyager 2 LECP $Z \geq 1$ ion spectra covering the period 1992-1994 (heliocentric distance 36.25 to $44.75 \mathrm{AU}$ ) are included in Giacalone et al. (1997). The flux at $40 \mathrm{keV}$ was $\sim 2 \times 10^{-2}\left(\mathrm{~cm}^{2} \mathrm{~s} \mathrm{sr} \mathrm{keV}\right)^{-1}$. Assuming that the flux varies with distance from the Sun in proportion to $1 / r^{2}$, this corresponds to $\sim 3.5 \times 10^{-3}\left(\mathrm{~cm}^{2} \mathrm{~s} \mathrm{sr} \mathrm{keV}\right)^{-1}$ at the distance of the termination shock. This is approximately similar to the flux measured by Voyager 1 upstream of the termination shock (period B in Fig. 2 
of Decker et al. 2006), but not in the foreshock flux enhancement region (period $\mathrm{C}$ ), and less than the heliosheath flux by about two orders of magnitude (Decker et al. 2006). If the ion flux in the heliosheath is approximately constant and if an $r^{-2}$ proportionality describes accurately the behaviour of the flux upstream from the shock, one can then estimate the contribution to the hydrogen ENA flux from the region inside the termination shock to be $\sim 0.3$ of the contribution from the heliosheath near the LISM apex direction. Our result is sensitive to the neutral hydrogen gas density profile, which decreases towards the Sun. We applied the hydrogen density profile and the heliosheath geometry provided by the Bonn model (Fahr et al. 2000). Note that the observed ENA flux is generated by the sunward-directed ion flux, which is less than the total ion flux in the case of pronounced field-aligned anisotropy, as observed inwards from the termination shock (Decker et al. 2005, 2007).

The above estimations are imprecise, but imply that the inner heliospheric contribution is not negligible compared to the ENA flux from the apex sector. If the inner heliospheric contribution is of the order of $\sim 0.3$ of the total ENA flux, the values of $N_{\mathrm{H}}$ and $L$ should be reduced by a factor $\sim 0.7$ compared to our result. This would reduce $N_{\mathrm{H}}$ to the value derived for model 6 (Table 2).

If Eq. (2) holds, Voyager 1 post-shock observations put a restriction on the inner heliospheric contribution to the ENA flux. The energetic ions inside the part of the heliosheath crossed by Voyager 1 until the end of 2007 ( $\sim 10 \mathrm{AU})$, already account for the hydrogen ENA flux that is equal to $\sim 0.25\left(n_{\mathrm{H}} /\left(0.1 \mathrm{~cm}^{-3}\right)\right.$ of the HSTOF data (but only half of that for the helium ENA). Even if we assume that the thickness of the heliosheath is close to the lower limit of model predictions that is $L=30 \mathrm{AU}$, the expected ENA flux from the heliosheath would be a fraction $\sim 0.75$ $\left(n_{\mathrm{H}} /\left(0.1 \mathrm{~cm}^{-3}\right)\right.$ of the HSTOF data.

\subsection{Time dependence}

The time dependence of the heliosheath geometry, in particular the termination shock motion, and its ion population can affect our estimation of $N_{\mathrm{H}}$ and $L$. However, we see two reasons for expecting that this effect is not large. First, the time periods of 2005-2007 (Voyager 1 heliosheath data) and 1996-1997 (significant part of the HSTOF ENA observations) are both close to solar minimum. The ion distributions in the heliosheath corresponding to Voyager 1 and to HSTOF observations may be similar. In (I), we also considered separately the 1996-1997 and the 1998-2003 time periods of the HSTOF observations. Assuming the same ion distributions, we found that the results for $N_{\mathrm{H}}$ were close to each other, suggesting that our results are not strongly sensitive to the observation time.

\section{Conclusions}

Assuming that the ion flux in the heliosheath is equal to the average spectrum measured by Voyager 1 (Eq. (2)), we have used the HSTOF ENA data, corresponding to the flux from the direction close to the apex of the Sun motion relative to the LISM (the "nose" of the heliosphere), for deriving the radial neutral hydrogen column density $N_{\mathrm{H}}$ in the sector of the heliosheath within $\pm 45^{\circ}$ ecliptic longitude from the LISM apex and in the two adjacent $90^{\circ}$ wide sectors. The result for the apex sector of $N_{\mathrm{H}}=(0.63 \pm 0.19) \times 10^{14} \mathrm{~cm}^{-2}$ corresponds to the thickness of the heliosheath $L=42 \pm 12$ AU at $n_{\mathrm{H}}=0.1 \mathrm{~cm}^{-3}$. For the adjacent sectors the results are more uncertain, because of larger angular distances from the region from which Voyager 1 ion data were obtained. We note that our earlier result of $N_{\mathrm{H}}=1.13 \times 10^{14} \mathrm{~cm}^{-2}$, Ref. (I) referred to the sector of the heliosheath larger than the sum of all three sectors considered here.

Comparison to numerical models of the heliosphere, which assume a presently preferred LISM neutral $\mathrm{H}$ density of $\sim 0.2 \mathrm{~cm}^{-3}$, imply that the HSTOF observations can be explained by assuming a high value (close to $0.1 \mathrm{~cm}^{-3}$ ) of proton density in the LISM, or another mechanism that can reduce the thickness of the heliosheath. The models used in the comparison are all simplified (time-independent and axisymmetric relative to the LISM apex direction). We have not discussed the implications of the crossing of the termination shock by Voyager 2, which indicate possible asymmetry in the heliosphere.

The ENA flux originating in the region inwards from the solar wind termination shock is probably not negligible in comparison with the heliosheath contribution. However, the high ion flux in the heliosheath observed by Voyager 1 up to the end of 2007 (after traversing $10 \mathrm{AU}$ in the heliosheath) already accounts for the ENA flux equal to $\sim 0.25$ of the HSTOF data.

Voyager $1 Z \geq 1$ ion data are consistent with the HSTOF hydrogen ENA spectra. The helium ENA flux measured by HSTOF is higher by about a factor 2 than the value expected on the basis of Voyager 1 ion data.

Acknowledgements. A.C. acknowledges support from Polish Ministry of Science and Higher Education grant 4T12E 00230.

\section{References}

Alexashov, D., \& Izmodenov, V. 2005, A\&A, 439, 1171

Baranov, V., \& Malama, Y. 1993, J. Geophys. Res., 98, 15157

Barnett, C. F., Hunter, H. T., Kirkpatrick, M. I., Alvarez, I., \& Phaneuf, R. A. 1990, Atomic Data for Fusion, Collisions of H, H2, He and Li Atoms and Ions with Atoms and Molecules, Oak Ridge Natl. Lab. Report, ORNL-6086VI, Oak Ridge, Tenn.

Czechowski, A., Hsieh, K. C., Hilchenbach, M., Kóta, J., \& Shaw, A. W. 2004, Adv. Space Res., 34, 104

Czechowski, A., Hilchenbach, M., \& Kallenbach, R. 2006a, in The Physics of the Heliospheric Boundaries, ed. V. V. Izmodenov, \& R. Kallenbach, ISSI Scientific Report SR-005, Chap. 10, 311

Czechowski, A., Hilchenbach, M., Hsieh, K. C., Kóta, J., \& Kallenbach, R. 2006b, ApJ, 647, L69 (I)

Czechowski, A., Hilchenbach, M., Hsieh, K. C., \& Kóta, J. 2007, in Proc. of the 30th International Cosmic Ray Conference, Merida, Mexico

Decker, R. B., Krimigis, S. M., Roelof, E. C., et al. 2005, Science, 309, 2020

Decker, R. B., Roelof, E. C., Krimigis, S. M., \& Hill, M. E. 2006, in Physics of the inner heliosheath, 5th IGPP International Astrophysics Conference, ed. J. Heerikhuisen, V. Florinski, G. P. Zank, \& N. V. Pogorelov, AIP Conf. Proc., 858,73

Decker, R. B., Krimigis, S. M., Roelof, E. C., \& Hill, M. E. 2007, in Turbulence and Nonlinear Processes in Astrophysical Plasmas, 6th Annual International Astrophysical Conference, ed. D. Shaikh, \& G. P. Zank, 197

Fahr, H. J., Kausch, T., \& Scherer, K. 2000, A\&A, 357, 268

Giacalone, J., Jokipii, J. R., Decker, R. B., et al. ApJ, 486, 471

Gloeckler, G. 1996, Space Sci. Rev., 78, 335

Gloeckler, G., \& Fisk, L. 2006, in Physics of the inner heliosheath, 5th IGPP International Astrophysics Conference, ed. J. Heerikhuisen, V. Florinski, G. P. Zank, \& N. V. Pogorelov, AIP Conf. Proc., 858, 153

Heerikhuisen, J., Florinski, V., \& Zank, G. P. 2006, J. Geophys. Res., 111, A06110

Hilchenbach, M., Hsieh, K. C., Hovestadt, D., et al. 1998, ApJ, 503, 916 Hilchenbach, M., Hsieh, K. C., Hovestadt, D., et al. 2001, in The Outer Heliosphere: The New Frontiers, COSPAR Colloq. Ser., 11, 273

Hilchenbach, M., Czechowski, A., Hsieh, K. C., \& Kallenbach, R. 2006, in Physics of the inner heliosheath, 5th IGPP International Astrophys. Conf., ed. J. Heerikhuisen, V. Florinski, G. P. Zank, \& N. V. Pogorelov, AIP Conf. Proc., 858, 276

Hilchenbach, M., Kallenbach, R., Czechowski, A., \& Hsieh, K. C. 2007, in Proceedings of the 30th International Cosmic Ray Conference, Merida, Mexico

Hovestadt, D., Hilchenbach, M., Bürgi, A., et al. 1995, Sol. Phys., 162, 441 Hsieh, K. C., Shih, K. L., Jokipii, J. R., \& Grzedzielski, S. 1992, ApJ, 393, 756 
Izmodenov, V., \& Alexashov, D. 2006, in Physics of the inner heliosheath, 5th IGPP International Astrophysics Conference, ed. J. Heerikhuisen, V. Florinski, G. P. Zank, \& N. V. Pogorelov, AIP Conf. Proc., 858, 14

Izmodenov, V. V., Malama, Y. G., \& Ruderman, M. S. 2008, Adv. Space Res., 41,318

Izmodenov, V. V., Geiss, J., Lallement, R., et al. 1999, JGR, 104, 4731

Kóta, J., Hsieh, K. C., Czechowski, A., Jokipii, J. R., \& Hilchenbach, M. 2001, J. Geophys. Res., 106, 24907

McComas, D., Allegrini, F., Bochsler, P., et al. 2004, In Physics of the Outer Heliosphere, Third Annual IGPP Conference, ed. V. Florinski, N. V. Pogorelov, \& G. P. Zank, AIP Conf. Proc., 719, 162

Müller, H. R., Frisch, P. C., Florinski, V., \& Zank, G. P. 2006, ApJ, 647, 1491

Müller, H. R., Florinski, V., Heerikhuisen, J., et al. 2007, A\&A, accepted

Pogorelov, N. V., Zank, G. P., \& Ogino, T. 2008a, Adv. Space Res., 41, 306
Pogorelov, N. V., Heerikhuisen, J., \& Zank, G. P. 2008b, ApJ, 675, L41

Quemerais, E., Bertaux, J.-L., Sandel, B. R., \& Lallement, R. 1994, A\&A, 290, 941

Scherer, K., \& Fahr, H.-J. 2003, Geophys. Res. Lett., 30, 17

Slavin, J. D., \& Frisch, P. C. 2007, A\&A, submitted [arXiv:0704.0657]

Stone, E. C., Cummings, A. C., McDonald, F. B., et al. 2005, Science, 309, 2017

Stone, E. C., Cummings, A. C., McDonald, F. B., et al. 2007, in Proc. of the 30th International Cosmic Ray Conference, Merida, Mexico

Washimi, H., Zank, G. P., Hu, Q., Tanaka, T., \& Munakata, K. 2007, ApJ, 670, L139

Witte, M. 2004, A\&A, 426, 835

Zank, G. P., Pauls, H. L., Williams, L. L., \& Hall, D. T. 1996, J. Geophys. Res., 101,21639 